\title{
Electronic and Vibrational Properties of Fe2NiAl and Co2NiAl Full Heusler Alloys: A First-Principles Comparison
}

This paper was downloaded from TechRxiv (https://www.techrxiv.org).

\section{LICENSE}

CC BY-NC-SA 4.0

SUBMISSION DATE / POSTED DATE

03-11-2021 / 05-11-2021

\section{CITATION}

Miroshkina, Olga; Sokolovskiy, Vladimir; Buchelnikov, Vasiliy; Gruner, Markus (2021): Electronic and Vibrational Properties of Fe2NiAl and Co2NiAl Full Heusler Alloys: A First-Principles Comparison. TechRxiv. Preprint. https://doi.org/10.36227/techrxiv.16917469.v1

DOI 


\title{
Electronic and Vibrational Properties of $\mathrm{Fe}_{2} \mathrm{NiAl}$ and $\mathrm{Co}_{2} \mathrm{NiAl}$ Full Heusler Alloys: A First-Principles Comparison
}

\author{
Olga N. Miroshkina ${ }^{1,2}$, Vladimir V. Sokolovskiy ${ }^{2}$, Vasiliy D. Buchelnikov' ${ }^{2}$, and Markus E. Gruner ${ }^{1}$ \\ ${ }^{1}$ Faculty of Physics and Center for Nanointegration, CENIDE, University of Duisburg-Essen, 47048 Duisburg, Germany \\ ${ }^{2}$ Faculty of Physics, Chelyabinsk State University, 454001 Chelyabinsk, Russia
}

\begin{abstract}
We provide a comparative first-principles investigation of the structural, electronic, vibrational and thermodynamic properties of the full Heusler compounds, cubic $\mathrm{Fe}_{2} \mathrm{NiAl}$ and tetragonally distorted $\mathrm{Co}_{2} \mathrm{NiAl}$. In both cases, we find the inverse $\mathrm{Heusler}$ structure to be in close competition with a layered arrangement of the elements, which breaks cubic symmetry and is accompanied by significant absolute values for the magnetocrystalline anisotropy energy. While for $\mathrm{Fe}_{2} \mathrm{NiAl}$ the layered arrangement is predicted as a new ground state, we show evidence that it might become dynamically stable around room temperatures also for $\mathrm{Co}_{2} \mathrm{NiAl}$. We identify in both systems subtle differences in the electronic and vibrational density of states between the different structures, which might be related to their particular stability.
\end{abstract}

Index Terms-Density functional theory, Heusler alloys, magnetocrystalline anisotropy, vibrational densities of states

\section{INTRODUCTION}

$\mathbf{P}$ ermanent magnets are of vital interest for modern industry since they are essential for the miniaturization of electronic devices, robotics, electrical vehicles, magnetic data storage and cooling technologies [1]-[10]. Nowadays, the most powerful commercial permanent magnets, such as $(\mathrm{Nd}, \mathrm{Pr})_{2} \mathrm{Fe}_{14} \mathrm{~B}$ and $\mathrm{Sm}_{2}(\mathrm{Co}, \mathrm{Cu}, \mathrm{Fe}, \mathrm{Zr})_{17}$, are based on rareearth elements, which are comparatively scarce and expensive. Therefore the search for rare-earth free permanent magnets is a central topic for modern materials science.

Among the distinguished candidates is tetratenite $\mathrm{L}_{0^{-}}$$\mathrm{FeNi}$, whose magnetocrystalline anisotropy energy (MAE) of $\approx 1.1-1.3 \mathrm{MJ} / \mathrm{m}^{3}$ [11]-[13] approaches the order of Nd-Fe-B. Tetratenite is mainly found in meteorites [14], [15] according to the rather low order-disorder phase transition temperature $\left(200-300{ }^{\circ} \mathrm{C}\right)$ leading to extremely slow atomic diffusion, which makes its synthesis very challenging on the large scale. However, $\mathrm{L}_{0}$-FeNi can be grown as a thin film [16]-[20], whereas other current strategies also include the effect of doping, interstitials, pressure, or simultaneous application of stress and magnetic field under an inert atmosphere on the order-disorder transformation [21]-[24].

In recent years, full Heusler alloys based on Ni [25], [26], $\mathrm{Fe}$ [26], [27], Co [26], [27], Rh, Au, and Mn [26] were considered for application as permanent magnets with a mediumrange energy product. The advantages of Heusler alloys are their comparatively low price and flexibility in properties, which can be tuned by structural ordering, composition and various defects. Fe- and Co-based Heusler alloys were recently studied by Matsushita et al. in terms of whether one may obtain a large MAE in alloys that adopt a low symmetry tetragonal structure [27]. Among 30 considered compositions, the authors found 15 , which undergo tetragonal distortion and have MAE from $-12 \mathrm{MJ} / \mathrm{m}^{3}\left(\mathrm{Co}_{2} \mathrm{PtAl}\right)$ to $+5.19 \mathrm{MJ} / \mathrm{m}^{3}\left(\mathrm{Fe}_{2} \mathrm{PtGe}\right)$. It was proposed in theoretical studies [27]-[30] that $\mathrm{Fe}_{2} \mathrm{NiAl}$

Corresponding author: O.N. Miroshkina (email: olga.miroshkina@unidue.de). possesses the inverse cubic structure (XA, $\mathrm{Hg}_{2} \mathrm{CuTi}$ prototype), which confirms part of the experimental results [28], [31], while conflicting with others. Yin et al. [32] performed direct synthesis calorimetry of standard formation enthalpies and found that $\mathrm{Fe}_{2} \mathrm{NiAl}$ crystallizes in a $\mathrm{B} 2$ structure in agreement with the high temperature phase diagram [33]. According to other studies, $\mathrm{Fe}-\mathrm{Ni}-\mathrm{Al}$ solid solutions are prone to a decomposition into two isomorphous body centered cubic $(b c c)$ phases, consisting of Fe-rich particles ( $\beta$-phase) and a weak-magnetic NiAl-based matrix ( $\beta_{2}$-phase) [34], [35] and as a result of the decomposition, a miscibility gap appears on the phase diagram at lower temperatures [33], [36], [37]. Nevertheless, a subsequent heat treatment is found to improve the hard magnetic properties of $\mathrm{Fe}-\mathrm{Ni}-\mathrm{Al}[38]-[40]$.

The theoretical discussion of these full Heusler compounds mainly concentrates on the regular (L2 $2_{1}$ ) and inverse (XA) structure. However, Neibecker et al. [41] recently suggested that layered crystal motives can be a strong competitor. This is corroborated by our recent efforts devoted to the firstprinciples study of structural stability and magnetic properties of these motifs in $(\mathrm{Fe}, \mathrm{Co})_{2} \mathrm{Ni}_{1+x} \mathrm{Al}_{1-x}$ [42], [43]. In particular, we identified the $\mathrm{T}^{p}$-layered structure with nearly cubic lattice parameters as a potential ground state for $\mathrm{Fe}$ based alloys at the stoichiometric composition and small $\mathrm{Ni}$ excess, whereas stoichiometric $\mathrm{Co}_{2} \mathrm{NiAl}$ has in turn an inverse tetragonal structure with $c / a=1.3$. Here $\mathrm{T}^{p}$ is close in energy, becoming more favorable with $\mathrm{Ni}$ excess [43]. In this work, we provide a comparative analysis of the electronic, vibrational and thermodynamic properties of stoichiometric $\mathrm{Fe}_{2} \mathrm{NiAl}$ and $\mathrm{Co}_{2} \mathrm{NiAl}$, which both were predicted to show a large MAE in their $\mathrm{T}^{p}$ structures: easy-axis and as large as $1.05 \mathrm{MJ} / \mathrm{m}^{3}$ for $\mathrm{Fe}_{2} \mathrm{NiAl}$ [42] and easy plane with a value of $-2.2 \mathrm{MJ} / \mathrm{m}^{3}$ for $\mathrm{Co}_{2} \mathrm{NiAl}[43]$.

\section{Details of the Calculations}

Ground state properties of $(\mathrm{Fe}, \mathrm{Co})_{2} \mathrm{NiAl}$ were calculated in the framework of density functional theory (DFT) using the plane-wave basis set and the projector augmented 

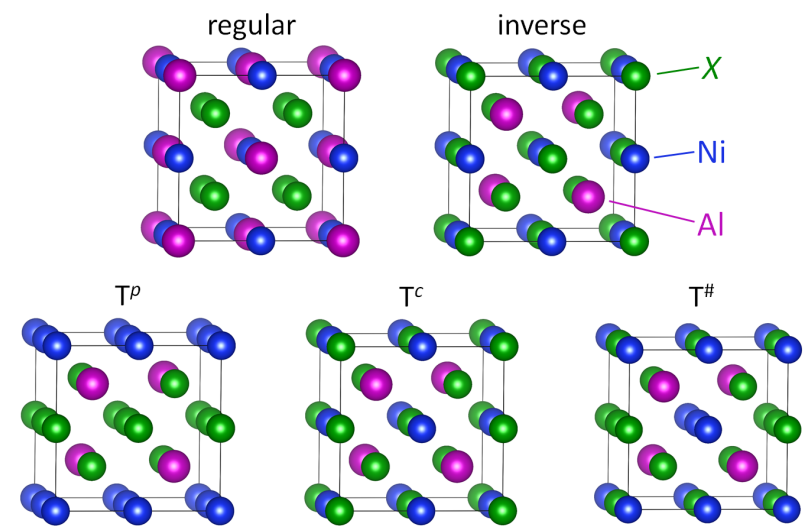

Fig. 1. Types of crystal ordering considered for $X_{2} \mathrm{NiAl}(X=\mathrm{Fe}$ and $\mathrm{Co})$. Regular L2 1 structure: two $X$ atoms locate at equivalent $8 c(1 / 4,1 / 4,1 / 4)$ and $(3 / 4,3 / 4,3 / 4)$ sites having identical environments, $\mathrm{Al}$ and $\mathrm{Ni}$ atoms occupy $4 a(0,0,0)$ and $4 b(1 / 2,1 / 2,1 / 2)$ positions, correspondingly. Inverse XA structure: two $X$ atoms are placed at non-equivalent $4 a(0,0,0)$ and $4 c(1 / 4$, $1 / 4,1 / 4)$ sites and have different environments, while $\mathrm{Ni}$ and $\mathrm{Al}$ are placed at $4 b(1 / 2,1 / 2,1 / 2)$ and $4 d(3 / 4,3 / 4,3 / 4)$ positions, respectively. $\mathrm{T}^{p}, \mathrm{~T}^{c}$, and $\mathrm{T}^{\#}$ can be constructed from the inverse structure by intermixing [41], [42].

wave (PAW) method as implemented in VASP [44]. The generalized gradient approximation (GGA) in the formulation of Perdew, Burke, and Ernzerhof (PBE) [45] was applied as exchange-correlation functional together with corresponding PAW potentials with the following valence states: $3 p^{6} 3 d^{7} 4 s^{1}$ for $\mathrm{Fe}, 3 d^{8} 4 s^{1}$ for $\mathrm{Co}, 3 p^{6} 3 d^{8} 4 s^{2}$ for $\mathrm{Ni}$, and $3 s^{2} 3 p^{1}$ for $\mathrm{Al}$. The energy cut-off for the plane wave basis set were set to $750 \mathrm{eV}$. The Brillouin zone integration was performed with the first order Methfessel-Paxton method using uniform Monkhorst-Pack $15 \times 15 \times 15 k$-point grid. We considered regular and inverse Heusler structures together with three types of layered atomic ordering, which are depicted in Fig. 1. All these structures were modeled using the 16-atom supercells. For the layered atomic arrangement taking place in $\mathrm{T}^{p}$ and $\mathrm{T}^{c}$ structures, the cubic symmetry is already broken even for $c / a=1$. In our calculations we considered the distortion along [001] ( $z$-axis) only $\left(E_{\text {tot }}=E_{c|| z}\right)$, concentrating on the ideal structures in the first step, whereas the lowest energy structures underwent an additional full optimization step with respect to lattice parameters and ionic positions. Vibrational density of states (VDOS) were calculated within the direct (force-constant) methods using supercells of up to 256 atoms with a cut-off energy of $500 \mathrm{eV}$. Employing the Phonopy [46] code, we obtained the VDOS and the lattice free energies in the harmonic approximation. For further details, we refer to Refs. [42] and [43].

\section{RESULTS}

\section{A. Structural and magnetic properties}

An overview of the energetic order of the structural motifs in Fig. 1 as a function of an additional tetragonal distortion $c / a$ is presented for both compositions in Fig. 2(a) and (c). One can see that the regular structure is comparatively far above the energy of the other structures for both $\mathrm{Fe}$ - and Co-based alloys, except for large tetragonal distortions. For $\mathrm{Fe}_{2} \mathrm{NiAl}$ (Fig. 2(a)), the $\mathrm{T}^{p}$ structure with almost equal lattice parameters $(c / a \approx$
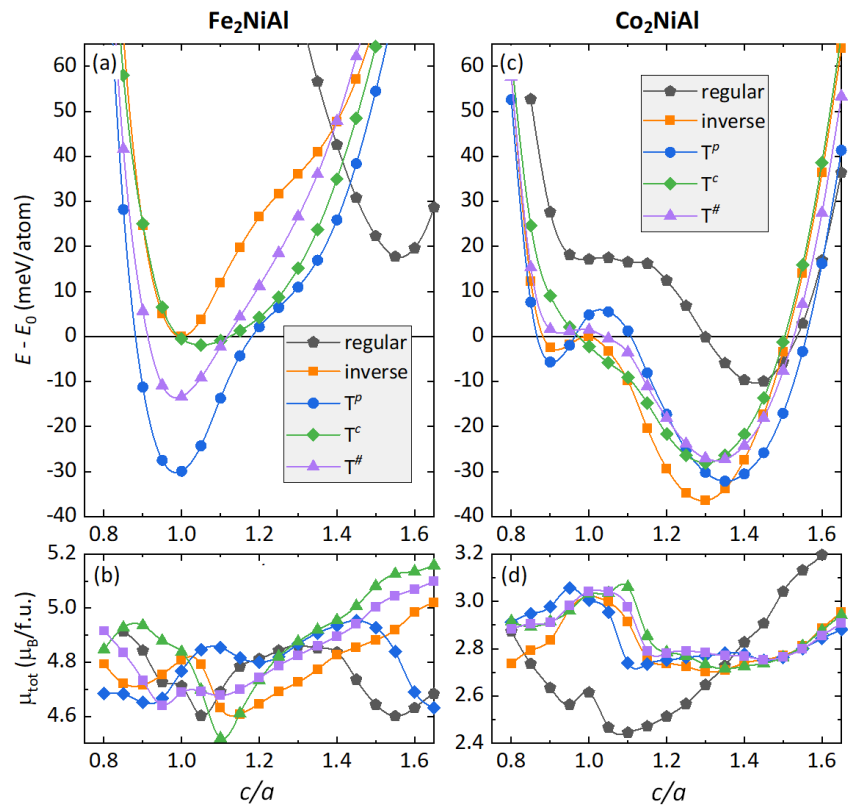

Fig. 2. Energy landscape and magnetization of $\mathrm{Fe}_{2} \mathrm{NiAl}$ (left panel) and $\mathrm{Co}_{2} \mathrm{NiAl}$ (right panel) as a function of the tetragonal distortion $c / a$. The energy $E_{0}$ of the respective inverse structure at $c / a=1$ is taken as reference for all curves. For $\mathrm{Fe}_{2} \mathrm{NiAl}$, a layered $\mathrm{T}^{p}$ structure with almost cubic lattice parameters is found to be the ground state, $\approx 36 \mathrm{meV} /$ atom below the inverse Heusler structure. The ground state of $\mathrm{Co}_{2} \mathrm{NiAl}$ is a tetragonally distorted inverse structure $(c / a=1.29)$, being in close competition with the $\mathrm{T}^{p}$ ordering with $c / a=1.35$. Data partially taken from Ref. [42], [43].

$0.99)$ is identified as a potential ground state structure, while other structures are at least $15 \mathrm{meV} / \mathrm{atom}$ (as in case of $\mathrm{T}^{\#}$ ) higher in energy. The energy curves of the inverse, $\mathrm{T}^{p}, \mathrm{~T}^{c}$, and $\mathrm{T}^{\#}$ structures have only one minimum corresponding to nearly cubic lattice parameters indicating the absence of martensitic phase transition for $\mathrm{Fe}_{2} \mathrm{NiAl}$. Contrary, a tetragonally distorted inverse (XA) structure is found be a ground state of $\mathrm{Co}_{2} \mathrm{NiAl}$ with $c / a=1.29$. However, the layered $\mathrm{T}^{p}$ structure with $c / a=1.35$ turns out to be a close competitor due to the small energy difference of $\approx 5 \mathrm{meV} /$ atom between them.

The magnetization per formula unit (f.u.) for the two compounds is shown in panels (b) and (d) of Fig. 2. $\mathrm{Fe}_{2} \mathrm{NiAl}$ offers a rather large magnetization in the order of $4.8 \mu_{\mathrm{B}} /$ f.u., being nearly the same for the inverse and $\mathrm{T}^{p}$ structure at $c / a=1$, while there seems no clear trend regarding its dependence on a tetragonal distortion. $\mathrm{Co}_{2} \mathrm{NiAl}$ has a considerably smaller moment of about $2.8 \mu_{\mathrm{B}} /$ f.u., as expected from the presence of two more $d$-electrons in the minority channel. Interestingly, the dependence of the magnetic moment curves on $c / a$ is rather similar for the inverse and $\mathrm{T}^{p}, \mathrm{~T}^{c}$, and $\mathrm{T}^{\#}$ structures as opposed to the regular $\left(\mathrm{L} 2_{1}\right)$ ordering.

All competitive structures under consideration are found to be stable with respect to decomposition into the primary phases of the constituting elements. This is demonstrated in Table $\mathrm{I}$ in terms of a negative formation energy $E_{\mathrm{form}}=$ $E_{\text {tot }}-\left(2 E_{X}+E_{\mathrm{Ni}}+E_{\mathrm{Al}}\right)$, where $E_{\text {tot }}$ is the total energy of $X_{2} \mathrm{NiAl}(X=\mathrm{Fe}$ and $\mathrm{Co}), E_{X}, E_{\mathrm{Ni}}$, and $E_{\mathrm{Al}}$ are the total energies of corresponding pure $X, \mathrm{Ni}$, and $\mathrm{Al}$ in their bulk phases. In absolute numbers, the stability of $\mathrm{Fe}_{2} \mathrm{NiAl}$ is 
TABLE I

STRUCTURAL PROPERTIES OF $\mathrm{FE}_{2} \mathrm{NIAL}_{\text {AND }} \mathrm{CO}_{2} \mathrm{NIAl}_{\text {AFTER FULL }}$ OPTIMIZATION OF THE LATTICE PARAMETERS AND IONIC POSITIONS. DATA TAKEN FROM REF. [42], [43].

\begin{tabular}{|c|c|c|c|c|c|}
\hline Alloy & Struct. & $\begin{array}{c}a \\
{[\AA]}\end{array}$ & $\begin{array}{c}c \\
{[\AA]}\end{array}$ & $c / a$ & $\begin{array}{c}E_{\text {form }} \\
{[\mathrm{eV} / \text { f.u. }}\end{array}$ \\
\hline \multirow{2}{*}{$\mathrm{Fe}_{2} \mathrm{NiAl}$} & $\mathrm{T}^{p}$ & 5.752 & 5.709 & 0.992 & -1.237 \\
\cline { 2 - 6 } & inverse & 5.739 & 5.738 & 1.000 & -1.089 \\
\hline \multirow{2}{*}{$\mathrm{Co}_{2} \mathrm{NiAl}$} & $\mathrm{T}^{p}$ & 5.141 & 6.914 & 1.345 & -0.775 \\
\cline { 2 - 6 } & inverse & 5.206 & 6.728 & 1.292 & -0.780 \\
\hline
\end{tabular}

significantly higher against the decomposition into the pure element phases as compared to $\mathrm{Co}_{2} \mathrm{NiAl}$.

\section{B. Electronic structure}

According to the fundamental difference in lattice symmetry (cubic vs. tetragonal) $\mathrm{Fe}_{2} \mathrm{NiAl}$ and $\mathrm{Co}_{2} \mathrm{NiAl}$ naturally exhibit substantial differences in their electronic density of states (DOS), see Fig. 3. The detailed comparison of the DOS between the inverse and $\mathrm{T}^{p}$ structure for each compound in Fig. 3(a) and (d), exhibits that in case of $\mathrm{Fe}_{2} \mathrm{NiAl}$ the reduced symmetry of the layered $\mathrm{T}^{p}$ with respect to the cubic XA arrangement also results in important modifications, notably the opening of a sharp pseudo-gap around $-1.2 \mathrm{eV}$ in the inverse structure, which is characterized by a tetrahedral coordination of $\mathrm{Al}$ with respect to the surrounding $\mathrm{Ni}$. In the element-resolved DOS in Fig. 3(c), we observe - apart from the overall strong hybridization between Fe and Ni- $d$ states an increased hybridization between Ni- $d$ and Al- $p$ as compared to the $\mathrm{T}^{p}$-case, which contributes to the rather sharp definition of the edges of the pseudo-gap. In the lower symmetry of the $\mathrm{T}^{p}$ structure, degenerate states tend to split up, which leads to the filling of the gap in Fig. 3(b) by redistributing states from $\approx-1.4 \mathrm{eV}$ in the majority and from $\approx-0.8 \mathrm{eV}$ and $\approx-2.2 \mathrm{eV}$ in the minority channel of inverse $\mathrm{Fe}_{2} \mathrm{NiAl}$. Eventually we also observe a widening of the deep minimum in the DOS around the Fermi-level in the $\mathrm{T}^{p}$ case, which might contribute to the particular stability of the structure although the value of the DOS directly at the Fermi level hardly differs between $\mathrm{T}^{p}$ and inverse Heusler structure.

In case of $\mathrm{Co}_{2} \mathrm{NiAl}$, the DOS is essentially almost for both $\mathrm{T}^{p}$ and inverse structures in the majority channel, while subtle but essential differences are visible for the spin-down states, see Fig. 3(d). This applies in particular to a sharp Fe- $d$-peak in the minority channel slightly below the Fermi-level, shown enlarged in the inset of Fig. 3(d). In the $\mathrm{T}^{p}$-structure, this peak moves slightly closer to the Fermi energy, which leads to a high density of states at the highest occupied level, which is a disadvantage in terms of the band energy and a destabilizing feature for the lattice. One can also note the splitting in the minority Ni DOS into two peaks at -0.9 to $-1.6 \mathrm{eV}$ in the inverse case in Fig. 3(f), which, however, does not lead to the formation of a pseudogap due to the lower symmetry of the structure, which causes additional broadening of the peaks. Similar to $\mathrm{Fe}_{2} \mathrm{NiAl}$, the minimum in between is filled in the $\mathrm{T}^{p}$ structure in Fig. 3(e), mainly with Ni states which originate around $-0.5 \mathrm{eV}$ in Fig. 3(f).
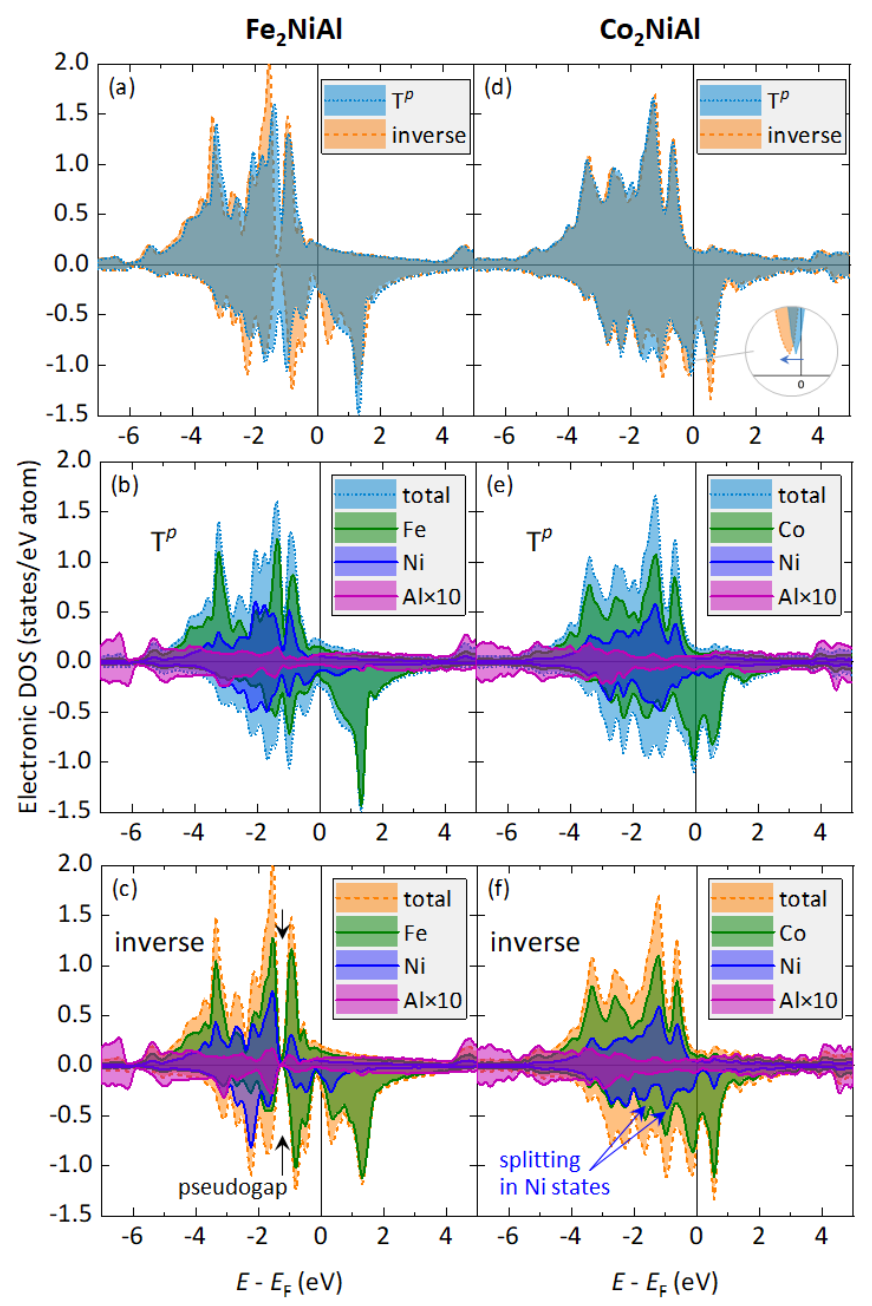

Fig. 3. Electronic DOS of $\mathrm{Fe}_{2} \mathrm{NiAl}$ (left column) and $\mathrm{Co}_{2} \mathrm{NiAl}$ (right column) in $\mathrm{T}^{p}$ and inverse structures. $\mathrm{Fe}_{2} \mathrm{NiAl}$ : In the $\mathrm{T}^{p}$ structure states move away from Fermi level for both occupied and unoccupied states in comparison with the inverse one, widening the minimum around $E_{r m F}$ in the minority channel while filling a pseudo-gap around $-1.2 \mathrm{eV} . \mathrm{Co}_{2} \mathrm{NiAl}$ : A sharp Copeak appears just below the Fermi level in the minority spin channel (see inset). This peak moves to the lower energies in the inverse structure.

\section{Vibrational and thermodynamic properties}

The VDOS as a fingerprint of the interatomic forces once again shows larger differences between the systems than between the structural motifs, as shown in Fig. 4(a) and (d). This is again to a large extend traced back to the significant tetragonal distortion of the Co-based system, which results in a characteristic broadening of the features, e.g., the sharp peak arising from vibrations of $\mathrm{Al}$ atoms around $10 \mathrm{THz}$, which occurs for $\mathrm{T}^{p}$ and inverse structure, see the element-resolved VDOS in Fig. 4(b,c,e,f). Nevertheless, Fig. 4(a) and (d) exhibit for both compositions subtle, but important differences in the VDOS between the two structural motifs. For $\mathrm{Fe}_{2} \mathrm{NiAl}$, a gaplike feature opens at $6.7 \mathrm{THz}$ in $\mathrm{T}^{p}$ structure, leading to a redistribution of states to a new peak around $7.5-8 \mathrm{THz}$ and towards the lower energy range between 2.5 and $4.5 \mathrm{eV}$, which is dominated by $\mathrm{Ni}$-vibrations in $\mathrm{T}^{p}$. In case of $\mathrm{Co}_{2} \mathrm{NiAl}$, we essentially observe a red-shift of the features in the energy range between 3 and $8 \mathrm{eV}$, which again results in a higher 

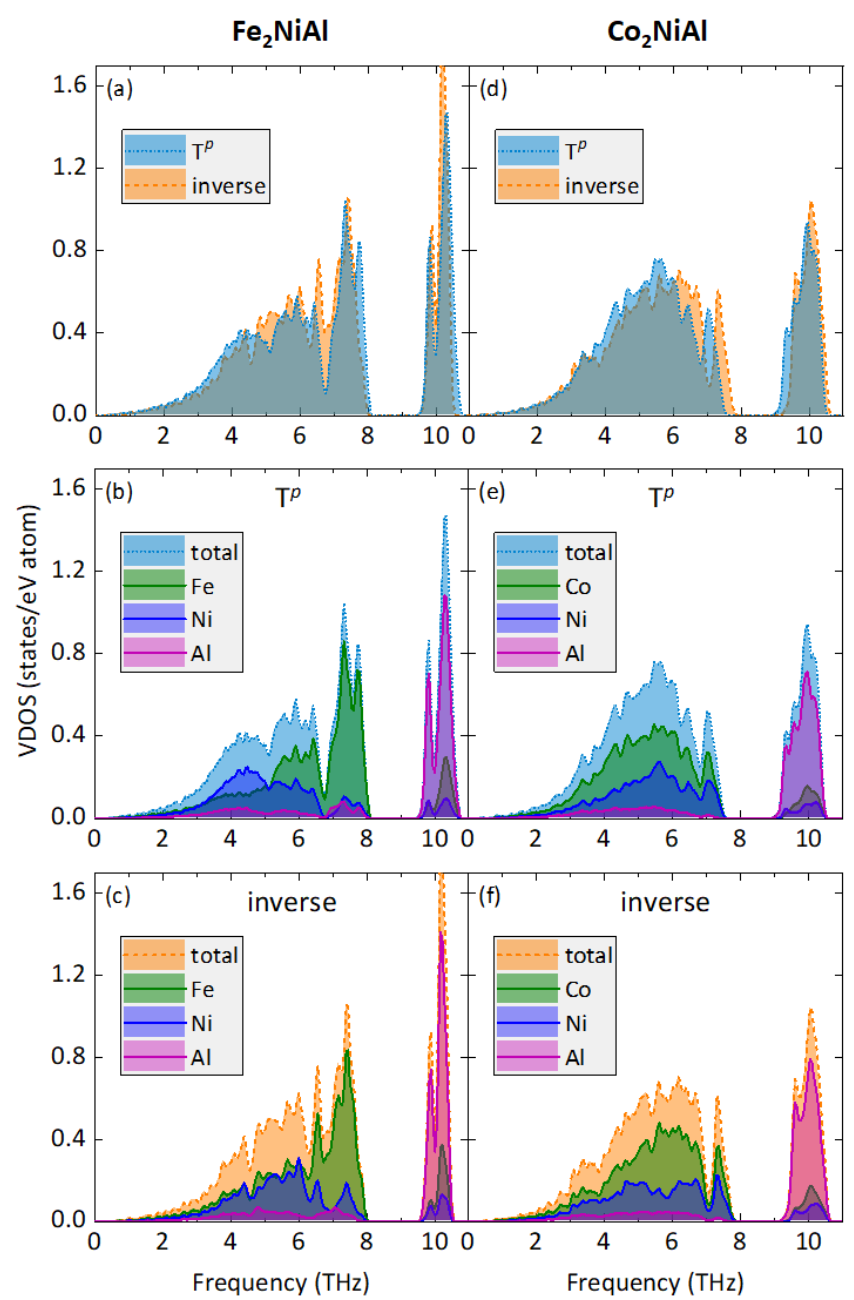

Fig. 4. Total and element-resolved vibrational DOS of $\mathrm{Fe}_{2} \mathrm{NiAl}$ (left column) and $\mathrm{Co}_{2} \mathrm{NiAl}$ (right column) in $\mathrm{T}^{p}$ and inverse structures. Comparison of the total VDOS for both compositions (top row) as well as the partial VDOS in $\mathrm{T}^{p}$ (middle row) and inverse (bottom row) are presented.

contribution of $\mathrm{T}^{p}$ in the lower energy range $<6 \mathrm{THz}$, arsing from an increase in the Co-VDOS.

Such an increase in density at low energies is indicative of a softening of both structures, which also affects thermodynamic quantities, in particular an increase the lattice entropy, which might further stabilize the $\mathrm{T}^{p}$ structure with increasing temperature. We therefore evaluated the free energy $F$ in the harmonic approximation of the structures at finite temperatures in a similar fashion as in [42], taking into account the vibrational contributions derived within the harmonic approximation from the VDOS in Fig. 4 and the electronic contributions from the DOS in Fig. 3 in terms of the Sommerfeld expansion, while the magnetic free energy is neglected according to the rather high Curie-temperatures in these compounds [42], [43]. The difference in the free energies $\Delta F(T)=F_{T^{p}}(T)-F_{\text {inverse }}(T)$ for both compounds as a function of temperature $T$ is presented in Fig. 5. For the Febased compound, the $\mathrm{T}^{p}$ structure possesses lowest free energy already at $T=0$ and improves its stability further in the entire temperature range. For $\mathrm{Co}_{2} \mathrm{NiAl}$, the inverse structure

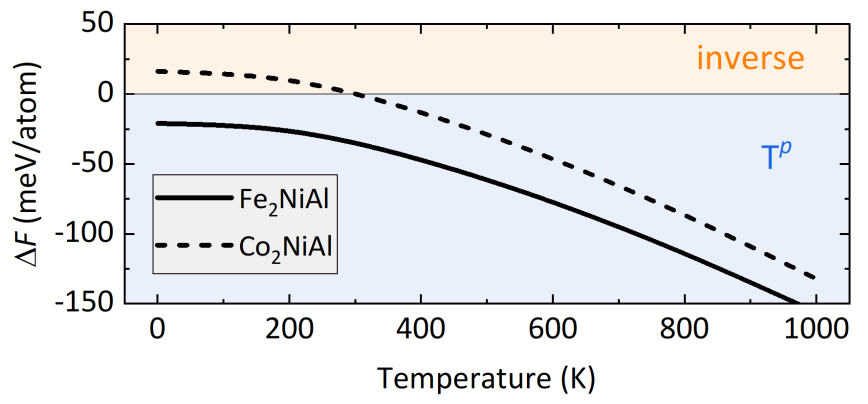

Fig. 5. Difference in free energy between $\mathrm{T}^{p}$ and inverse structures of $\mathrm{Fe}_{2} \mathrm{NiAl}$ and $\mathrm{Co}_{2} \mathrm{NiAl}$, respectively, as a function of temperature. The layered ordering is preferred for $\mathrm{Fe}_{2} \mathrm{NiAl}$ in the entire temperature range, whereas the energetic order changes around room temperature for $\mathrm{Co}_{2} \mathrm{NiAl}$.

is favored below $300 \mathrm{~K}$, while above the red-shifted VDOS causes a preference for $\mathrm{T}^{p}$, which becomes more and more stable with increasing temperatures. This indicates a possibility for the synthesis of $\mathrm{T}^{p}$ structures in both compounds under suitable annealing conditions.

\section{Conclusions}

In this work, we compare the inverse Heusler structures with the recently suggested layered $\mathrm{T}^{p}$ ordering in stoichiometric $\mathrm{Fe}_{2} \mathrm{NiAl}$ and $\mathrm{Co}_{2} \mathrm{NiAl}$ compounds in the framework of density functional theory. The layered atomic ordering turns out to be a promising candidate for the ground state of $\mathrm{Fe}_{2} \mathrm{NiAl}$, while it becomes stable at ambient condition in $\mathrm{Co}_{2} \mathrm{NiAl}$ due to its advantage in lattice entropy. The stability of $\mathrm{T}^{p}$ ordering in the two compounds is connected to subtle differences between characteristic features in the electronic and vibrational DOS.

Based on these findings, we expect that the layered $\mathrm{T}^{p}$ ordering may be a strong competitor to the inverse (XA) structure also in other Heusler compounds with a similar valence electron concentration, regardless whether the system undergoes a martensitic transition to a tetragonal ground state. Due to the broken symmetry, layered $\mathrm{T}^{p}-\mathrm{Fe}_{2} \mathrm{NiAl}$ possesses a large MAE of the same order as tetrataenite $\mathrm{FeNi}$ - even in a phase with cubic lattice parameters, which makes a $\mathrm{T}^{p_{-}}$ type layered order an interesting feature for rare-earth free permanent magnets in Heusler-type compounds.

\section{ACKNOWLEDGMENT}

This work was supported by Deutsche Forschungsgemeinschaft (DFG, German Research Foundation) within TRR 270 (Project-ID 405553726), Russian Science Foundation (RSF) project No. 17-72-20022 (structural and magnetic properties of $\mathrm{Fe}_{2} \mathrm{NiAl}$ ), and the Ministry of Science and Higher Education of the Russian Federation within the framework of the Russian State Assignment under contract No. 075-00992-21-00 (structural and magnetic properties of $\mathrm{Co}_{2} \mathrm{NiAl}$ ). Vibrational properties calculations were performed on the MagnitUDE high performance computing system of the University of DuisburgEssen (DFG INST 20876/209-1 and 20876/243-1 FUGG). 


\section{REFERENCES}

[1] P. Campbell, "Permanent magnet materials and their application," 1996.

[2] D. Goll and H. Kronmüller, "High-performance permanent magnets," Naturwissenschaften, vol. 87, no. 10, pp. 423-438, 2000.

[3] B. Bochenkov and S. Lutz, "A review of modern materials of permanent magnets," in Proceedings. The 8th Russian-Korean International Symposium on Science and Technology, 2004. KORUS 2004., vol. 1. IEEE, 2004, pp. 201-203.

[4] R. Skomski and J. Coey, "Magnetic anisotropy—how much is enough for a permanent magnet?" Scr. Mater., vol. 112, pp. 3-8, 2016.

[5] R. Skomski, Permanent Magnets: History, Current Research, and Outlook. Cham: Springer International Publishing, 2016, pp. 359-395.

[6] K. Hono and H. Sepehri-Amin, "Prospect for HRE-free high coercivity Nd-Fe-B permanent magnets," Scr. Mater., vol. 151, pp. 6-13, 2018.

[7] K. Skokov and O. Gutfleisch, "Heavy rare earth free, free rare earth and rare earth free magnets-vision and reality," Scr. Mater., vol. 154, pp. 289-294, 2018.

[8] J. Mohapatra and J. P. Liu, Rare-Earth-free permanent magnets: the past and future. Elsevier, 2018, vol. 27, pp. 1-57.

[9] A. Kovacs, J. Fischbacher, M. Gusenbauer, H. Oezelt, H. C. Herper, O. Y. Vekilova, P. Nieves, S. Arapan, and T. Schrefl, "Computational design of rare-earth reduced permanent magnets," Engineering, vol. 6, no. 2, pp. 148-153, 2020.

[10] J. Coey, "Perspective and prospects for rare earth permanent magnets," Engineering, vol. 6, no. 2, pp. 119-131, 2020.

[11] J. Paulevé, A. Chamberod, K. Krebs, and A. Bourret, "Magnetization curves of $\mathrm{Fe}-\mathrm{Ni}(50-50)$ single crystals ordered by neutron irradiation with an applied magnetic field," Journal of Applied Physics, vol. 39, no. 2, pp. 989-990, 1968.

[12] M. Mizuguchi, T. Kojima, M. Kotsugi, T. Koganezawa, K. Osaka, and K. Takanashi, "Artificial fabrication and order parameter estimation of $\mathrm{L} 1_{0}$-ordered FeNi thin film grown on a AuNi buffer layer," J. Magn. Soc. Jpn., vol. 35, no. 4, pp. 370-373, 2011.

[13] E. Poirier, F. E. Pinkerton, R. Kubic, R. K. Mishra, N. Bordeaux, A. Mubarok, L. H. Lewis, J. I. Goldstein, R. Skomski, and K. Barmak, "Intrinsic magnetic properties of $\mathrm{L} 1_{0} \mathrm{FeNi}$ obtained from meteorite NWA 6259," Journal of Applied Physics, vol. 117, no. 17, p. 17E318, 2015.

[14] J. Cui, M. Kramer, L. Zhou, F. Liu, A. Gabay, G. Hadjipanayis, B. Balasubramanian, and D. Sellmyer, "Current progress and future challenges in rare-earth-free permanent magnets," Acta Materialia, vol. 158, pp. 118-137, 2018.

[15] L. H. Lewis, A. Mubarok, E. Poirier, N. Bordeaux, P. Manchanda, A. Kashyap, R. Skomski, J. Goldstein, F. Pinkerton, R. Mishra et al., "Inspired by nature: investigating tetrataenite for permanent magnet applications," J. Phys. Condens. Mat., vol. 26, no. 6, p. 064213, 2014.

[16] E. Lima Jr and V. Drago, "A new process to produce ordered $\mathrm{Fe}_{50} \mathrm{Ni}_{50}$ tetrataenite," Phys. Stat. Sol. (A), vol. 187, no. 1, pp. 119-124, 2001.

[17] T. Shima, M. Okamura, S. Mitani, and K. Takanashi, "Structure and magnetic properties for $\mathrm{L}_{0}$-ordered $\mathrm{FeNi}$ films prepared by alternate monatomic layer deposition," J. Magn. Magn. Mater, vol. 310, no. 2, pp. 2213-2214, 2007.

[18] A. Makino, P. Sharma, K. Sato, A. Takeuchi, Y. Zhang, and K. Takenaka, "Artificially produced rare-earth free cosmic magnet," Sci. Rep., vol. 5, no. 1, pp. 1-8, 2015.

[19] S. Goto, H. Kura, E. Watanabe, Y. Hayashi, H. Yanagihara, Y. Shimada, M. Mizuguchi, K. Takanashi, and E. Kita, "Synthesis of single-phase $\mathrm{L} 1_{0}$-FeNi magnet powder by nitrogen insertion and topotactic extraction," Sci. Rep., vol. 7, no. 1, pp. 1-7, 2017.

[20] G. Giannopoulos, G. Barucca, A. Kaidatzis, V. Psycharis, R. Salikhov, M. Farle, E. Koutsouflakis, D. Niarchos, A. Mehta, M. Scuderi et al., "L1 $1_{0}$-FeNi films on Au-Cu-Ni buffer-layer: a high-throughput combinatorial study," Sci. Rep., vol. 8, no. 1, pp. 1-9, 2018.

[21] L.-Y. Tian, H. Levämäki, O. Eriksson, K. Kokko, Á. Nagy, E. K Délczeg-Czirják, and L. Vitos, "Density functional theory description of the order-disorder transformation in Fe-Ni," Sci. Rep., vol. 9, no. 1, pp. 1-7, 2019.

[22] L.-Y. Tian, O. Eriksson, and L. Vitos, "Pressure effect on the orderdisorder transformation in $L 1_{0}$ FeNi," Sci. Rep., vol. 10, no. 1, pp. 1-7, 2020.

[23] N. Maât, I. McDonald, R. Barua, B. Lejeune, X. Zhang, G. Stephen, A. Fisher, D. Heiman, I. Soldatov, R. Schäfer et al., "Creating, probing and confirming tetragonality in bulk FeNi alloys," Acta Materialia, vol. 196, pp. 776-789, 2020.
[24] L.-Y. Tian, O. Gutfleisch, O. Eriksson, and L. Vitos, "Alloying effect on the order-disorder transformation in tetragonal FeNi," Sci. Rep., vol. 11, no. 1, pp. 1-9, 2021.

[25] H. C. Herper, "Ni-based Heusler compounds: How to tune the magnetocrystalline anisotropy," Phys. Rev. B, vol. 98, no. 1, p. 014411, 2018.

[26] Q. Gao, I. Opahle, O. Gutfleisch, and H. Zhang, "Designing rare-earth free permanent magnets in Heusler alloys via interstitial doping," Acta Mater., vol. 186, pp. 355-362, 2020.

[27] Y. Matsushita, G. Madjarova, J. Dewhurst, S. Shallcross, C. Felser, S. Sharma, and E. Gross, "Large magnetocrystalline anisotropy in tetragonally distorted Heuslers: a systematic study," J. Phys. D: Appl. Phys., vol. 50, no. 9, p. 095002, 2017.

[28] Y. Zhang, W. Wang, H. Zhang, E. Liu, R. Ma, and G. Wu, "Structure and magnetic properties of $\mathrm{Fe}_{2} \mathrm{Ni} Z(Z=\mathrm{Al}, \mathrm{Ga}, \mathrm{Si}$ and $\mathrm{Ge})$ Heusler alloys," Physica B: Condens. Matter, vol. 420, pp. 86-89, 2013.

[29] D. C. Gupta and I. H. Bhat, "Full-potential study of $\mathrm{Fe}_{2} \mathrm{Ni} Z(Z=\mathrm{Al}, \mathrm{Si}, \mathrm{Ga}, \mathrm{Ge}), " \quad$ Mater. Chem. Phys., vol. 146, no. 3, pp. 303-312, 2014

[30] F. Dahmane, Y. Mogulkoc, B. Doumi, A. Tadjer, R. Khenata, S. B. Omran, D. Rai, G. Murtaza, and D. Varshney, "Structural, electronic and magnetic properties of $\mathrm{Fe}_{2}$-based full Heusler alloys: A first principle study," J. Magn. Magn. Mater., vol. 407, pp. 167-174, 2016.

[31] E. S. Popiel, W. Zarek, and M. Tuszyński, "Mossbauer study of the Heusler-type $\mathrm{Fe}_{2} M \mathrm{Al}$ compounds for $M=\mathrm{V}, \mathrm{Cr}, \mathrm{Fe}, \mathrm{Co}, \mathrm{Ni}$," $N u k$ leonika, vol. 49, pp. 49-52, 2004.

[32] M. Yin, P. Nash, and S. Chen, "Enthalpies of formation of selected $\mathrm{Fe}_{2} Y Z$ Heusler compounds," Intermetallics, vol. 57, pp. 34-40, 2015.

[33] L. Zhang, J. Wang, Y. Du, R. Hu, P. Nash, X.-G. Lu, and C. Jiang, "Thermodynamic properties of the Al-Fe-Ni system acquired via a hybrid approach combining calorimetry, first-principles and CALPHAD," Acta Mater., vol. 57, no. 18, pp. 5324-5341, 2009.

[34] A. Bradley and A. Taylor, "An x-ray investigation of the cause of high coercivity in iron-nickel-aluminium alloys," Nature, vol. 140, no. 3554, pp. 1012-1013, 1937.

[35] V. Menushenkov, M. Gorshenkov, E. Savchenko, I. Shchetinin, and A. Savchenko, "Formation of high-coercivity state in $\mathrm{Fe}_{2} \mathrm{NiAl}$ alloy during decomposition of solid solution under quenching from liquid state and subsequent annealing," Heat Treat. Met., vol. 59, no. 7-8, pp. 518-523, 2017.

[36] S. M. Hao, T. Takayama, K. Ishida, and T. Nishizawa, "Miscibility gap in Fe-Ni-Al and Fe-Ni-Al-Co systems," Metall. Trans. A, vol. 15, no. 10, pp. 1819-1828, 1984

[37] L. Zhang, Y. Du, H. Xu, C. Tang, H. Chen, and W. Zhang, "Phase equilibria of the $\mathrm{Al}-\mathrm{Fe}-\mathrm{Ni}$ system at $850^{\circ} \mathrm{C}$ and $627^{\circ} \mathrm{C}, " \mathrm{~J}$. Alloys Compd., vol. 454, no. 1-2, pp. 129-135, 2008.

[38] K. Buschow and F. de Boer, Physics of Magnetism and Magnetic Materials. Springer US, 2007.

[39] V. Menushenkov, M. Gorshenkov, D. Zhukov, E. Savchenko, and M. Zheleznyi, "Peculiarities of the spinodal decomposition and magnetic properties in melt-spun $\mathrm{Fe}_{2} \mathrm{NiAl}$ alloy during aging," Mater. Lett., vol. 152, pp. 68-71, 2015.

[40] V. Menushenkov, M. Gorshenkov, I. Shchetinin, A. Savchenko, E. Savchenko, and D. Zhukov, "Evolution of the microstructure and magnetic properties of as-cast and melt spun $\mathrm{Fe}_{2} \mathrm{NiAl}$ alloy during aging," J. Magn. Magn. Mater., vol. 390, pp. 40-49, 2015.

[41] P. Neibecker, M. E. Gruner, X. Xu, R. Kainuma, W. Petry, R. Pentcheva, and M. Leitner, "Ordering tendencies and electronic properties in quaternary Heusler derivatives," Phys. Rev. B, vol. 96, no. 16, p. 165131, 2017.

[42] V. Sokolovskiy, O. Miroshkina, V. Buchelnikov, and M. Gruner, "Impact of local arrangement of $\mathrm{Fe}$ and $\mathrm{Ni}$ in $\mathrm{Fe}-\mathrm{Ni}-\mathrm{Al}$ Heusler alloys on the phase stability and magnetocrystalline anisotropy," arXiv:2109.12005 [cond-mat.mtrl-sci], 2021.

[43] V. Sokolovskiy, O. Miroshkina, A. Sanosyan, D. Baigutlin, V. Buchelnikov, and M. Gruner, "Magnetic and structural properties of Co-Ni- $Z$ ( $Z=\mathrm{Al}, \mathrm{Ga}, \mathrm{In}, \mathrm{Sn})$ Heusler alloys: effect of structural motives and chemical disorder," J. Magn. Magn. Mater. (accepted), 2021.

[44] G. Kresse and J. Furthmüller, "Efficient iterative schemes for ab initio total-energy calculations using a plane-wave basis set," Phys. Rev. B, vol. 54, pp. 11 169-11 186, Oct 1996.

[45] J. Perdew, K. Burke, and M. Ernzerhof, "Generalized Gradient Approximation Made Simple," Phys. Rev. Lett., vol. 77, p. 3865, 1996.

[46] A. Togo and I. Tanaka, "First principles phonon calculations in materials science," Scr. Mater., vol. 108, pp. 1-5, Nov 2015. 